\title{
Global optimization of protein-peptide docking by a filling function method
}

\author{
Francesco Lampariello, Giampaolo Liuzzi \\ Istituto di Analisi dei Sistemi ed Informatica (IASI) \\ CNR \\ Rome, Italy \\ giampaolo.liuzzi@iasi.cnr.it
}

\begin{abstract}
Molecular docking programs play a crucial role in drug design and development. In recent years, much attention has been devoted to the protein-peptide docking problem in which docking of a flexible peptide with a known protein is sought. In this work we present a new docking algorithm which is based on the use of a filling function method for continuos constrained global optimization. Indeed, the protein-peptide docking position is sought by minimizing the conformational potential energy subject to constraints necessary to maintain the primary sequence of the given peptide. The resulting global optimization problem is difficult mainly for two reasons. First, the problem is large scale in constrained global optimization; second, the energy function is multivariate non-convex so that it has many local minima. The method is based on the device of modifying the original objective function once a local minimum has been attained by adding to it a filling term. This allows the overall algorithm to escape from local minima thus, ultimately, giving the algorithm ability to explore large regions in the peptide conformational space. We present numerical results on a set of benchmark docking pairs and comparison with the well-known software package for molecular docking PacthDock.
\end{abstract}

Keywords-Protein-peptide docking; potential reduction; continuous global optimization

\section{Introduction}

In this paper we address the problem of docking small peptide molecules, for example, drug candidates, onto a given protein model. Molecular docking programs play a crucial role in drug design and development. In recent years, much attention has been devoted to this problem where docking of a flexible peptide with a known protein is sought. We consider a docking algorithm based on the use of a filling function method for continuos unconstrained global optimization [1].

The correct protein-peptide docking position is obtained by minimizing the function representing the total potential energy according to a specific mathematical model. In order to preserve the primary sequence of the given peptide it is necessary to take into account some constraints on the problem variables, and then we construct the Lagrangian of the original problem. The resulting optimization problem has two main features; it is a large-scale one in constrained global optimization, and the total potential energy function has many local minima. Once a local minimum has been found, the method modifies the original objective function by adding to it a filling term. This allows the algorithm to escape from the local minimum so that it may explore large regions in the search space.

The approaches proposed in the literature are based on the minimization of successive approximations of some potential energy function, obtained by introducing some suitable parameters (see, e.g. [2], [3]) in order to simplify the single minimization step. Moreover, these methods determine the docking between the peptide and a prefixed small part of the protein, namely the prefixed receptor or binding site. Most of these methods allow for receptor flexibility [4], [3], [2]. The minimization process is based on a multi-start strategy that uses the steepest descent or conjugate gradient methods as local minimization tools.

\section{The Energy Model}

As regards the mathematical model employed, we assume that the protein tertiary structure and the peptide primary residue sequence are known and we denote by $\mathrm{N}$ and $M$ the number of peptide and protein residues, respectively.

In order to find the docking pocket and position of the peptide onto the protein, we consider that peptide is flexible in such a way that all its atoms have three degrees of freedom. Moreover, the given peptide primary residue sequence is not modified while calculating the docking position by means of suitable simple nonlinear constraints. Let

$$
E_{L J}^{i j}=4 \epsilon_{i j}\left[\left(\frac{\sigma_{i j}}{r_{i j}}\right)^{12}-\left(\frac{\sigma_{i j}}{r_{i j}}\right)^{6}\right], E_{C}^{i j}=\frac{q_{i} q_{j}}{4 \pi \epsilon_{0} r_{i j}}
$$

be the Lennard-Jones and Coulomb potentials, respectively. They represent the interaction between protein atom $i$ and peptide atom $j$ and $r_{i j}$ denotes their distance. Hence, we consider the following total potential energy function [3],

$$
E(r)=\sum\left(E_{L J}^{i j}+E_{C}^{i j}\right)
$$


where the summation is calculated over all pairs $(N \times M)$ of atoms.

\section{Global Optimization Approach}

Here we consider the problem of finding the docking position of a given peptide by taking into account all the given protein atoms, so that the binding site is not prefixed, but directly determined by the algorithm. In order to avoid that the peptide primary sequence is modified, we consider the following constraints between the carbon alpha atoms of the peptide residues:

$$
r_{i, i+1}^{c}=3.8, \forall i=1, \ldots, N-1
$$

and

$$
r_{i, k}^{c} \geq 2, \forall i=1, \ldots, N-2, k=i+2, \ldots, N .
$$

Therefore, our algorithm computes the final docking position by searching for a global minimum of $E(r)$ subject to (2) and (3).

For the sake of clarity, let us consider the general constrained nonlinear global optimization problem

$$
\begin{aligned}
& \text { globminf }(x) \\
& \text { s.t. } x \in F,
\end{aligned}
$$

where $x \in R^{n}$,

$$
\begin{array}{r}
F=\left\{x \in \Re^{n}: g(x) \leq 0\right\}, \\
f: \Re^{n} \rightarrow \Re, g: \Re^{n} \rightarrow \Re^{m} .
\end{array}
$$

Let us assume, as usual, that $f$ and $g$ are continuously differentiable functions and that $f(x)$ is radially unbounded on the feasible set. Now, let $L(x, \lambda)=f(x)+\lambda^{T} g(x)$, with $\lambda \in \mathfrak{R}^{m}$, be the Lagrangian function for Problem (4). A pair $\left(x^{i}, \lambda^{i}\right)$ is a Karush-Kuhn-Tucker (KKT) pair if [5]

$$
g\left(x^{i}\right)=0, \quad g\left(x^{i}\right)^{T} \lambda^{i}=0, \quad \lambda^{i} \geq 0, \nabla L\left(x^{i}, \lambda^{i}\right)=0 .
$$

Under some mild regularity assumptions on the constraint functions, if $x^{i}$ is a local minimum point of Problem (4), we have [5]:

Proposition 1: Let $x^{i}$ be a local minimum of Problem (4). Then, a vector $\lambda^{i} \in R^{m}$ of KKT multipliers exists such that $\left(x^{i}, \lambda^{i}\right)$ is a KKT pair. Furthermore, the hessian of the Lagrangian function $\nabla_{x}^{2} L\left(x^{*}, \lambda^{*}\right)$ is positive definite.

Finally, we assume that a local optimization routine is available, namely, a routine that, given an arbitrary starting point $\mathrm{x}$, provides a local minimum of Problem (4). More in particular, as local optimization engine we use the augmented Lagrangian algorithm ALGENCAN [6], [7].

\section{A. The filling function technique}

Starting from a point randomly chosen in $\Re^{n}$, let $x_{0}$ be the local minimum reached by the local optimization routine and $\lambda_{0} \in \Re^{m}$ be the vector of corresponding KKT multipliers.

In order to search over the various minima for finding that corresponding to the lowest $\mathrm{f}$ value, we have to escape the basin of attraction of the current minimum $x_{0}$. To do this, we consider the quadratic model of Problem (4) in $x_{0}$, that is,

$$
q\left(x ; x_{0}\right)=\frac{1}{2}\left(x-x_{0}\right)^{\top} H_{0}\left(x-x_{0}\right)
$$

where $H_{0}$ is the Hessian of the Lagrangian function for Problem (4) computed at $x_{0}$ Then, we construct the following gaussian-based function

$$
\varphi\left(x ; x_{0}\right)=\frac{\beta}{1-\exp \left(-\alpha q\left(x ; x_{0}\right)\right)}-\beta
$$

where ${ }^{\alpha}$ and $\beta$ are positive scalars, and we consider the new function obtained by adding to the original function $f(x)$

$$
\tilde{f}\left(x ; x_{0}\right)=f(x)+\varphi\left(x ; x_{0}\right) .
$$

Since

$$
q\left(x_{0} ; x_{0}\right)=0
$$

we have

$$
\varphi\left(x_{0} ; x_{0}\right)=+\infty
$$

so that

$$
\hat{f}\left(x_{0} ; x_{0}\right)=+\infty \text {. }
$$

Moreover, since $\varphi\left(x ; x_{0}\right) 0$, for allx,

$$
\hat{f}\left(x ; x_{0}\right)>f(x), \quad \forall x .
$$

Then,

$$
\varphi\left(x ; x_{0}\right) \rightarrow 0, \text { and } \hat{f}\left(x ; x_{0}\right) \rightarrow f(x)
$$

as $\| x-x_{0} / \longmapsto+\infty$, that is far from $x_{0}$ in any direction.

Therefore, function (8) is substantially the original function modified only locally, i.e., obtained by "filling" $f$ within a neighborhood of $x_{0}$, whose aplitude can be varied by taking different values of the parameter $\alpha$.

Thus, by applying the local search routine to starting from a point near $x_{0}$, we reach a minimum point $\hat{x}_{\text {which }}$ can not be $x_{0}$ and which is not a minimum of $f$.

Now, by reapplying the local search to the original 
function $f(x)$ starting from $\hat{x}$, either the same point $x_{0}$ is reached, or a new minimum point is found.

In the first case, the value of $\alpha$ is not sufficiently low to escape the basin of attraction of $x_{0}$, and a lower $\alpha$ value is needed.

\section{B. The global optimization procedure}

The global optimization algorithm can be summarized in the following scheme.

\section{Global Optimization Algorithm (GOAL)}

Data. $s_{0}, \Delta s, \epsilon$, integers $p \geq 10$, and $R \geq 1$.

Step 0. Set $r=1$ and $\lambda=1$.

Step 1. Generate at random a point $\tilde{x} \in \Re^{n}$, compute $\tilde{f}^{\tilde{}}(x)$ and apply the local search routine for minimizing $f(x)$. Let $x_{0}$ be the minimum reached; set $x^{(r)}=x_{0}$.

Step 2. If $r=1$, set $f_{\text {min }}=f\left(x_{0}\right), x_{\text {min }}=x_{0}$, else, if $\quad\left|k x_{0}-x^{(k)}\right| k \epsilon, \quad$ for an index $k=1, \ldots, r-1$, set $\lambda=\lambda+1$, and if $\lambda \leq r$ go to Step 1; otherwise STOP.

Step 3. Set ${ }^{i=1}$ and $f_{l}=f^{\tilde{r}}(x)$.

Step 4. Compute the parameters (see (9) below), where $s=s_{0}+(i-1) \Delta s$.

Step

5.

from $x_{0}+\Delta x$, minimize $\hat{f}\left(x ; x_{0}\right)$ Let $\hat{x}_{i}$ be the minimum reached; minimize $f(x)$ starting from $\hat{x}_{i}$, and let $x_{i}$ be the minimum obtained.

Step

If $\quad\left|k x_{i}-x^{(k)}\right| \ngtr \epsilon$, for all $k=1, \ldots, r, \quad$ and $f_{l}<f\left(x_{i}\right)$, set $f_{l}=f\left(x_{i}\right)$, and $x_{l}=x_{i}$. Set $i=i+1$, and if $i \leq p$ go to Step 4 .

Step

If $f_{l}<f_{\text {min }}$, set $f_{\text {min }}=f_{l}$, and $x_{\text {min }}=x_{l}$.

7. $r<R$, set $r=r+1$; otherwise STOP.

$f_{l}<f(x)$, then set $x^{(r)}=x_{l}, x_{0}=x_{l}$, and go to Step 3;

otherwise, set $\lambda=1$ and go to Step 1 .

The value of $\epsilon$ which is the lowest distance between two minimum points over which they are considered distinct, should be chosen taking into account the stopping criterion of the local search routine.

Note that the procedure, differently from the classical multistart algorithm, terminates after the prefixed maximum number $R$ of restarts have been performed, regardless the number of initial points chosen at random. Thus, it is even possible that a new restarting minimum point is always found, i.e., from Step 6 the algorithm never returns to Step 1, so that only one initial random point is employed.

As regards the parameters $\alpha$ and $\beta$ in function (7), in order to establish the amplitude of the neighborhood of a minimum xo where the function $\mathrm{f}$ is filled, we take

$$
\alpha=\frac{9}{s^{2},} \quad \beta=\frac{\bar{\nabla}_{\pi}}{3} s,
$$

where $s$ is the distance from $x_{0}$. Thus, the prefixed distance $s$ corresponds to $3 \sigma$, where $\sigma$ is the standard deviation of the Gaussian function in (6). The procedure employs $p$ increasing values of the prefixed distance $s$, and correspondingly the function $f$ is filled within neighborhoods of $x_{0}$ larger and larger.

\section{Preliminary Results}

In this Section we present some preliminary numerical results and comparison with the well-known software package for molecular docking PatchDock [8], [9] in terms of computed protein-peptide dockings for 23 proteinpeptide pairs. We implemented our method in double precision Fortran 90 and run the code on an Intel core 2 duo procesenr writh $\triangle$ SR $\mathrm{R}$ am under $\mathrm{I}$ inuv nneratio evetem by using $\Delta s=s_{0}=0.1, \epsilon=10^{-3}, p=25$ and $R=50$.

We applied our method to proteins in complex with specific ligands which are taken from the PDB [10]. The obtained results are summarized in Table I where we report, for each protein-peptide pair: (a) the name of the PDB entry; (b) the number of residues, NPE and MPR, composing, respectively, the peptide and the protein; (c) the avarage RMSD (root mean square distance) in Angstrom (A) between the computed and the known peptide docking position obtained by running our code (GOAL) and PatchDock from ten randomly chosen starting peptide positions.

In the table we use boldface to highlight a success. As it can be seen, GOAL outperforms PatchDock on 19 out of 23 pairs.

TABLEI DISTANCES BETWEEN COMPUTED AND KNOWN POSITIONS

\begin{tabular}{|l|r|r|c|c|}
\hline & & & GOAL & PatchDock \\
\hline PDB name & NPE & MPR & RMSD & RMSD \\
\hline 1A30(A) & 3 & 99 & $\mathbf{9 . 7 2 7 0 7 8 4}$ & 15.969474 \\
1A30(B) & 3 & 99 & $\mathbf{1 0 . 2 6 5 1 1 7}$ & 15.969474 \\
1 AWQ & 6 & 58 & $\mathbf{8 . 6 9 2 8 1 2 9}$ & 24.954113 \\
1 I31 & 6 & 97 & $\mathbf{7 . 7 2 7 2 0 1 0}$ & 22.708910 \\
1G3F & 9 & 69 & 6.9038916 & $\mathbf{6 . 3 2 7 4 4 5}$ \\
1VWG & 8 & 47 & 8.6765423 & $\mathbf{7 . 6 0 2 9 3 5}$ \\
1 AB9 & 10 & 51 & $\mathbf{7 . 9 9 8 3 4 8 7}$ & 11.081827 \\
1BE9 & 5 & 35 & $\mathbf{6 . 5 0 8 4 5 2 9}$ & 32.219646 \\
1GUX & 9 & 142 & $\mathbf{9 . 7 2 6 4 2 9 9}$ & 32.202145 \\
\hline
\end{tabular}




\begin{tabular}{|l|r|r|l|c|}
\hline 2FIB & 4 & 77 & $\mathbf{6 . 9 5 8 9 9 5 3}$ & 9.243341 \\
1BXL & 16 & 95 & 5.6981535 & $\mathbf{3 . 9 3 1 7 5 1}$ \\
1DUZ & 9 & 250 & $\mathbf{4 . 5 0 3 7 2 5 1}$ & 25.406809 \\
1F95 & 9 & 32 & 7.3566127 & $\mathbf{3 . 9 8 5 8 0 6}$ \\
1YCQ & 11 & 33 & $\mathbf{5 . 9 8 0 4 4 5 9}$ & 24.322502 \\
1EG4 & 13 & 33 & $\mathbf{7 . 1 2 5 9 4 1 3}$ & 18.821020 \\
1IO6 & 10 & 59 & $\mathbf{2 . 7 6 7 5 0 0 9}$ & 5.870293 \\
1CKA & 9 & 66 & $\mathbf{5 . 8 0 8 0 6 5 9}$ & 59.454960 \\
1ELW & 8 & 28 & $\mathbf{4 . 2 6 3 9 1 0 8}$ & 23.193996 \\
2SEB & 12 & 219 & $\mathbf{1 0 . 6 7 1 9 7 2}$ & 31.363981 \\
1CE1 & 8 & 85 & $\mathbf{1 9 . 1 3 4 8 5 1}$ & 28.343372 \\
1PAU & 4 & 52 & $\mathbf{1 7 . 1 2 5 2 5 4}$ & 52.701431 \\
1EVH & 5 & 47 & $\mathbf{1 1 . 5 4 4 4 2 9}$ & 15.931898 \\
1BC5 & 5 & 98 & $\mathbf{1 8 . 7 6 4 6 9 6}$ & 37.939034 \\
\hline
\end{tabular}

As we can see, the method is able to guess the peptide docking position with a maximum r.m.s.d of 10.7A (for 2SEB), meaning that the region of correct binding has been located by the method. Then, it is reasonable to think that the computed position can be further refined to the exact position, by allowing for receptor flexibility, applying, for instance, a proviously proposed tool (e.g. DynaDOCK [3], AutoDOCK [11], FDS [2]) which is the subject of ongoing work.

\section{Conclusions}

In the paper we present a global optimization method based on a gaussian filling function applied to the proteinpeptide docking problem. Indeed, the problem is defined as that of minimizing the potential energy function (1) subject to some constraints necessary to preserve the peptide primary sequence. The preliminary numerical results obtained, in terms of r.m.s.d. values, show viability of the proposed approach.

We are currently trying to improve the approach by considering more accurate potential energy functions. Moreover, we are attempting to exploit to a greater extent the chemical-physical properties of the atoms composing the peptide and the given protein, and to use some refinement tools for improving the computed final docking position.

\section{REFERENCES}

[1] F. Lampariello, "A filling function method for continuos unconstrained global optimization: application to morse clusters," IASI-CNR, Tech. Rep. R.615, 2004.

[2] R. D. Taylor, P. J. Jewsbury, and J. W. Essex, "FDS:Flexible ligand and receptor docking with a continuum solvent model and soft-core energy function," Journal of Computational Chemistry, vol. 24, pp. 1637-1656, 2003.

[3] I. Antes, "Dynadock: A new molecular dynamics-based algorithm for proteinpeptide docking including receptor flexibility," Proteins: Structure, Function and Bioinformatics, vol. 78, pp. 1084-1104, 2010.

[4] J. Apostolakis, A. Pluckthun, and A. Caflisch, "Docking small ligands in flexible binding sites," Journal of
Computational Chemistry, vol. 19, pp. 21-37, 1998.

[5] D. Bertsekas, Nonlinear Programming. Massachusetts, USA: Athena Scientific, 1999.

[6] R. Andreani, E. Birgin, J. Martinez, and M. Schuverdt, "On augmented lagrangian methods with general lower-level constraints," SIAM Journal on Optimization, vol. 18, pp. 1286-1309, 2007.

[7] —, "Augmented lagrangian methods under the constant positive linear dependence constraint qualification," Mathematical Programming, vol. 111, pp. 5-32, 2008.

[8] D. Duhovny, R. Nussinov, and H. Wolfson, "Efficient unbound docking of rigid molecules," in Proceedings of the 2'nd Workshop on Algorithms in Bioinformatics(WABI) Rome, Italy, ser. Lecture Notes in Computer Science 2452, Gusfield et al., Ed. Springer Verlag, 2002, pp. 185-200.

[9] D. Schneidman-Duhovny, Y. Inbar, R. Nussinov, and H. Wolfson, "Patchdock and symmdock: servers for rigid and symmetric docking," Nucleic Acids Research, vol. 33, pp. 363-367, 2005.

[10] H. Berman, J. Westbrook, Z. Feng, G. Gilliland, T. Bhat, H. Weissig, I. Shindyalov, and P. Bourne, "The protein data bank," Nucleic Acids Research, vol. 28, pp. 235242,2000

[11] G. Morris, D. Goodsell, R. Halliday, R. Huey, W. Hart, R. Belew, and A. Olson, "Automated docking using a lamarckian genetic algorithm and an empirical binding free energy function," Journal of Computational Chemistry, vol. 19, pp. 1639-1662, 1998. 